\title{
El bienestar social y su relación con la salud y la calidad de vida en personas adultas mayores
}

\author{
Social welfare and its relationship with health and quality of life in elderly people \\ Mynor Rodríguez Hernández ${ }^{1}$
}

Fecha de recepción: 6-12-2018

Fecha de aprobación: 10-9-2019

\begin{abstract}
Resumen
El acelerado crecimiento de la población adulta mayor debe ser considerado prioridad por las organizaciones responsables de este sector social. Envejecer es un proceso natural, el cual las personas deben afrontar, así como aceptar los cambios fisiológicos, mentales y sociales que trae. Estos cambios suelen afectar la salud de estas personas y con ello, su calidad de vida. Por lo tanto, este estudio se enfoca en determinar la relación que existe entre el bienestar social, la salud y la calidad de vida de la persona adulta mayor que participa en programas institucionalizados en Costa Rica. Un total de 138 participantes, mujeres y hombres, con edades entre 60 y 86 años ( $\bar{X}=67,94$ años $\pm 5,26)$, de grupos organizados de San Ramón, Costa Rica. La información se recopiló a través del cuestionario SF-36, con el objetivo de conocer la calidad de vida, salud y bienestar social de los adultos entrevistados, las respuestas se analizaron siguiendo la Escala de Ansiedad y Depresión de Goldberg. El análisis de los resultados muestra que la salud, el bienestar social y la calidad de vida son aceptables en este grupo. Existe correspondencia entre la Integración Social y las dimensiones de Salud General, Vitalidad, Función Social y Salud Mental ( $<<0.01)$, con la Función Física ( $<<0.05$ ). La dimensión Contribución Social se relacionó con los dominios de Función Física, Salud General, Vitalidad, Función Social, Salud Mental ( $\mathrm{p}<0.01)$ y con Transición de Salud ( $<<0.05)$. Por su parte la Actualización Social se correlacionó positivamente con la Función Física, Salud Mental y Transición de Salud ( $<<0.01$ ) y con la Salud General, Vitalidad y Función Social ( $<<0.05$ ), la salud percibida parcialmente fue más baja en edades avanzadas. El bienestar social es un determinante de la calidad de vida y la salud de las personas adultas mayores, por lo que, implementar estrategias de intervención que incluyan técnicas de socialización podría generar mejoras sustanciales en la salud y la calidad de vida de este grupo poblacional.

Palabras clave: Persona adulta mayor, calidad de vida, bienestar social, salud, envejecimiento.
\end{abstract}

\footnotetext{
1 Doctor en Ciencias del Movimiento Humano. Universidad de Costa Rica, Sede de Occidente. Departamento de Ciencias de la Educación.
} San Ramón, Alajuela, Costa Rica. Correo electrónico: mynorgrh@gmail.com 


\begin{abstract}
The worldwide increment in elderly population should be considered as a high priority by the organizations that serve these groups. The aging process involves a series of physiological, mental and social changes that affect the quality of life and health of these people. Therefore, this study aimed to determine the relationship between the social well-being, health and quality of life of the elderly person who participates in institutionalized programs. A total of 138 participants, women and men, aged between 60 and 86 years $(\bar{X}=67.94$ years \pm 5.26 ), from organized groups of San Ramon Costa Rica, participated in this study. The information was collected through the $\mathrm{SF}_{3} 6$ questionnaire to determine the perceived quality of life, the perception of health through the Goldberg scale and social welfare through the social well-being scale. The results show that perceived health, quality of life and social well-being are acceptable in this group. There were correlations between Social Integration and the dimensions of General Health, Vitality, Social Function and Mental Health ( $\mathrm{p}<0.01)$ and with Physical Function (p <0.05). The Social Contribution dimension was related to the domains of Physical Function, General Health, Vitality, Social Function, Mental Health (p <0.01) and Health Transition ( $\mathrm{p}<0.05)$. On the other hand, Social Update positively correlated with the Physical Function, Mental Health and Health Transition ( $\mathrm{p}<0.01$ ) and with General Health, Vitality and Social Function ( $\mathrm{p}<0.05)$, the perceived health was lower in advanced ages. Social well-being is a determinant of the quality of life and health of the elderly, so implementing intervention strategies that include socialization techniques could generate substantial improvements in the health and quality of life of this population group.
\end{abstract}

Key words: Senior person, quality of life, social well-being, health, aging.

\title{
I. Introducción
}

La población mundial muestra un incremento en la tasa poblacional de personas adultas mayores, debido a fenómenos relacionados con una mejor atención médica y al incremento en la expectativa de vida, aunado a ello, se espera que una gran parte de esta población formará parte del grupo de personas mayores de 60 años en el mundo. Sin embargo, hay que considerar que esta población es la más vulnerable a las enfermedades y situaciones de deterioro de la calidad de vida, esta última comprendida como la auto-percepción de su propia vida, en el contexto cultural, valores, metas, expectativas, estándares y preocupaciones y del que forman parte elementos como la salud física, el estado psicológico, el nivel de independencia, las relaciones sociales de la persona y su relación con el ambiente que le rodea (Rodríguez, 2008). El proceso de envejecimiento conlleva cambios que se manifiestan paulatinamente y que tienen que ver con el mantenimiento de la calidad de vida tales como: la disminución en el funcionamiento de los órganos o sistemas, la pérdida de autonomía y la pérdida de estándares sociales relacionados con ser personas útiles y productivas (Carbonell et al., 2009, Vera 2015, Ortiz y Castro, 2009).
El envejecimiento es un proceso cuya calidad está directamente relacionada con la forma como la persona satisface sus necesidades diarias (Collazo et al., 2017) y se ve mejorado en la medida en que esas necesidades sean cubiertas y haya un estado de independencia funcional (Ferreira et al., 2017). En estudios relacionados con esta temática se expone los efectos de la dependencia. Por ejemplo, se reporta que en Estados Unidos el 40\% de las personas mayores de 65 años tienen limitaciones para realizar las actividades de la vida diaria, lo que implica el aumento en los gastos y en la necesidad de contar con personas especializadas para su atención (Collins et al., 2004).

La disminución de la autonomía está relacionada directamente con la calidad de vida que a su vez es determinada por el estado psico-social y físico de la persona, los que pueden limitar la vida en términos cuantitativos y cualitativos en detrimento de la calidad de vida (Vera, 2013; Schwartzmann, 2003). Según reportes, las personas adultas mayores son capaces de adaptarse a estos cambios de vida, replanteándose sus metas y desafíos, adecuándolas a sus nuevas capacidades sociales, 
psicológicas y físicas, lo que disminuye la frustración al fracaso cuando no se puede llegar a concretar las metas propuestas de la misma forma en que se hacía antes (Ortiz y Castro, 2009).

Otro componente que parece tener impacto directo sobre la vida de la persona adulta mayor es el bienestar social, el cual se asocia directamente con la salud y la calidad de vida. Se dice que un adecuado bienestar social produce efectos positivos reduciendo el riesgo de mortalidad, de desarrollar enfermedades cardiovasculares, mortalidad por cáncer y el declive funcional (Levasseur et al., 2004). En contraparte, una calidad de vida deteriorada, también afecta el funcionamiento social, ya que induce al aislamiento y las personas se retiran de las actividades sociales normales afectando su estado emocional y de salud (Unger et al., 1999).

Aunado a ello, el aislamiento social y la falta de apoyo social son probablemente factores mediadores de estrés agudo y crónico que afecta a factores biológicos y de comportamiento, lo que produce efectos negativos a largo plazo sobre la salud, aumenta la susceptibilidad a la enfermedad, disminuye la calidad de vida e incrementa el riesgo de muerte temprana. El insuficiente apoyo social y el aislamiento son factores productores de estrés, lo que se asocia con efectos negativos sobre el sistema inmune, los procesos metabólicos y el sistema cardiovascular, así como los comportamientos relacionados con la salud (White et al., 2009). En un estudio longitudinal con personas adultas mayores y sus respectivas familias, se concluye que el adulto mayor necesita en su vida cotidiana paz, tranquilidad, protección familiar, digna y amorosa, sin perder su derecho a la libre expresión, comunicación y decisión. Además, necesita satisfacer las necesidades de seguridad, estabilidad, posesión, pertenencia y autorrealización (Vera, 2013).

Por su parte, según la Organización Panamericana de la Salud (OPS, 2004) la soledad y la inactividad son enemigos acérrimos de la vejez. Cuando la persona mayor se queda sola, sobre todo sin sus seres queridos, la tristeza la embarga y las ganas de vivir disminuyen. La ausencia de apoyo social origina carencias de todo tipo que incluso puede llevar a una muerte temprana
(Morales, 2015). El apoyo social y la calidad de vida se complementan, lo que significa que mejores niveles de apoyo social se asocian con mejor calidad de vida y menor presencia de sintomatología depresiva (Gallegos et al., 2009). La participación en las relaciones interpersonales, las responsabilidades, la aptitud y la recreación se asocia a una buena calidad de vida; además, la participación social puede contribuir significativamente a mejorar la calidad de vida de los adultos mayores con dependencia física (Levasseur et al., 2004).

Siguiendo esta línea, en un estudio realizado en Estados Unidos el $17 \%$ de la población adulta mayor reportó estar insatisfecha con el apoyo emocional, el 5\% indicó no tener ningún apoyo emocional, y el 59,6\% si recibió apoyo emocional. Mientras que el $62 \%$ mencionó tener buenos lazos sociales y el 32\% dijeron tener al menos un número mínimo de amigos con los cuales compartir, lo preocupante es que el 3,7\% no tenía ningún apoyo social, con un bienestar social, salud y calidad de vida deteriorados (White et al., 2009). Adicional a ello, las personas con un menor bienestar social auto-reportado, presentan mayores problemas de salud y su funcionalidad física se veía comprometida (Penninx et al., 1999). El bienestar social es un importante predictor del estado de salud de las personas adultas mayores, y tiene una fuerte relación con la calidad de vida. Las personas adultas mayores que tienen más y mejor apoyo social y muestran mejores resultados en el bienestar social, gozan de una mejor calidad de vida.

Por lo tanto, es fundamental que el adulto mayor pueda contar no sólo con el apoyo de los miembros de su familia, sino también con amigos o vecinos. Los adultos mayores que tienen una red social amplia mantienen un nivel de bienestar mayor en relación con los adultos que mantienen vínculos muy reducidos. Se señala que las relaciones unidireccionales de apoyo social dentro de los adultos mayores, generan sentimientos de soledad e insatisfacción, de modo que el mejor intercambio para el adulto mayor parece ser aquel en donde da y recibe apoyo (Salinas et al., 2008). 
Según lo planteado anteriormente, el bienestar social, la salud y la calidad de vida parecen ser aspectos estrechamente relacionados en la vida cotidiana de la persona adulta mayor. Por lo tanto, el objetivo de esta investigación se centró en determinar la relación existente entre el bienestar social, la salud y la calidad de vida de las personas adultas mayores que asisten a programas en donde pueden participar realizando rutinas de actividad física, baile, caminata y otras actividades lúdicas.

\section{Metodología}

Esta investigación se fundamenta en un estudio retrospectivo para observar y analizar la correlación existente entre las variables bienestar social, salud y calidad de vida en personas adultas mayores de grupos organizados para la práctica de actividades lúdicas de forma regular.

En este estudio se utilizó una muestra no probabilística en la que participaron un total de 138 personas adultas mayores, con edades entre 60 y 86 años, entre ellos 120 mujeres y 18 hombres con un promedio de edad de 67,94 \pm 5,26 años. Los participantes se ubicaron principalmente en los diferentes grupos organizados de actividad física de San Ramón de la Universidad de Costa Rica (9o personas de un total de 100) y la Caja Costarricense de Seguro Social (48 personas de un total de 52). Los criterios de inclusión y exclusión para participar en este estudio indicaron que las personas debían tener una edad igual o mayor a 60 años, que participaran en algún proyecto organizado en la comunidad donde realizaran actividad física regularmente, que fueran personas funcionales físicamente (participación activa en un programa por al menos 6 meses previos) y que fueran capaces de completar los cuestionarios y demás evaluaciones de forma individual y satisfactoria.

Los participantes de este estudio se ubicaron en los grupos organizados de personas adultas mayores de la comunidad. Una vez determinados los grupos meta, se procedió a obtener los permisos respectivos para acceder a las personas en cada grupo. Se contactó a cada persona por medio de correo electrónico, teléfono y de forma presencial. Se inscribió a todas las personas interesadas en participar en una lista de espera y se procedió a realizar las citas de trabajo para la recolección de la información. Se cumplió con los procedimientos de explicación y aclaración de la información y con la firma del consentimiento informado. Luego se aplicó el cuestionario calidad de vida SF-36, el cuestionario de percepción de salud de Goldberg y el cuestionario de bienestar social.

Las personas participantes se agruparon de acuerdo con su edad en cuatro subgrupos, como se explica a continuación: a) 6o-64 años, b) 65-69 años, c) 70-74 años, d) 75 años-más.

\subsection{Instrumentos}

Cuestionario de Calidad de Vida SF-36: Este cuestionario mide la percepción de calidad de vida relacionada con la salud, consta de 36 ítems situados en 8 dimensiones: función física, rol físico, dolor corporal, salud general, vitalidad, función social, rol emocional y salud mental. Cada dimensión tiene un puntaje mínimo de o y máximo de 100 puntos y la consistencia interna de todas las escalas es muy alta con un alfa de Cronbach de $\alpha=0,84$ a 0,95 (López et al., 2003).

Escala de bienestar social: Esta escala la desarrolló Corey Keyes y analiza mediante un cuestionario la valoración que cada persona hace de sí misma acerca de las circunstancias y funcionamiento dentro de la sociedad. Esta escala consta de 25 ítems que valoran integración social $\alpha=0.69$, la aceptación social $\alpha=0.83$, la contribución social $\alpha=0.70$, la actualización social $\alpha$ $=0.79$ y la coherencia social $\alpha=0.68$. El valor más bajo que se puede obtener en cada una de estas dimensiones es de 1 reflejando un bienestar social deficiente y el mayor es de 5 e indica un excelente bienestar psicológico (Blanco y Díaz, 2005).

Percepción de Salud de "Goldberg”: Este es un cuestionario de auto-reporte de salud general elaborado por David Goldberg y que indica la valoración que hace de sí mismo cada persona respecto a su estado de bienestar general, especialmente en lo que se refiere a ciertos estados emocionales. Se reporta un valor de alfa de Cronbach $\alpha=0,89$. Esta escala está compuesta por 12 ítems y el resultado se contabiliza de o hasta 36 puntos, donde o 
corresponde a una muy mala salud general y 36 puntos corresponde a una excelente salud percibida (García, 1999).

\section{Análisis estadístico}

Se utilizó estadística descriptiva (promedios y desviaciones estándar) para cada variable dependiente, así como correlaciones de "Pearson". Además, se utilizó el análisis de varianza (ANOVA) de una vía para determinar las variaciones por grupo de edad en cuanto a la percepción del bienestar social, la salud y la calidad de vida. Se utilizó un criterio de significancia de p $<0.05$ y los análisis fueron realizados mediante el paquete estadístico para las ciencias sociales "SPSS" versión 24.o.

\section{Resultados}

Un total de 138 personas, mujeres y hombres, con edades entre 60 y 86 años ( $\bar{X}=67,94$ años $\pm 5,26$ ), de grupos organizados de San Ramón participaron de este estudio. A continuación, se detallan los resultados encontrados en cuanto a las variables calidad de vida, salud percibida y bienestar social.

Calidad de vida

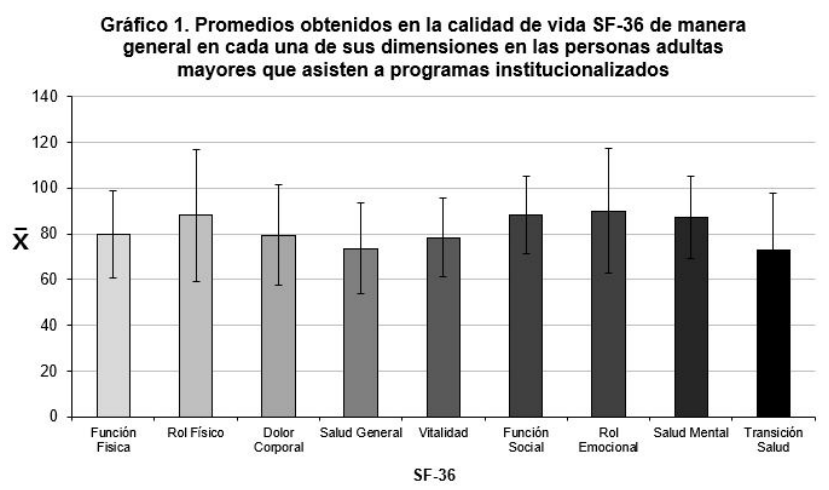

El gráfico 1 describe los valores promedio registrados mediante el uso del SF-36 en sus respectivas dimensiones. La calidad de vida percibida promedió valores superiores a 7o. Por ejemplo, la dimensión de "transición de salud" alcanzó un promedio de 72,8 , siendo el puntaje promedio más bajo y en contraparte el "rol emocional" obtuvo un promedio de 90,1.
Tabla 1. Promedios obtenidos en el protocolo de calidad de vida SF-36 por rango de edad en cada una de sus dimensiones en las personas adultas mayores que asisten a programas institucionalizados.

\begin{tabular}{|c|c|c|c|c|}
\hline & 60-64 años & $65-69$ años & $70-74$ años & 75 y más años \\
\hline & $\mathrm{N}=39$ & $\mathrm{~N}=48$ & $\mathrm{~N}=35$ & $\mathrm{~N}=16$ \\
\hline & $\overline{\mathbf{X}}+$ DS & $\overline{\mathbf{X}}+\mathrm{DS}$ & $\overline{\mathbf{X}}+\mathrm{DS}$ & $\overline{\mathbf{X}}+$ DS \\
\hline Función Física (FF) & $84,23 \pm 16,4^{4}$ & $82,42 \pm 17,5^{\prime \prime}$ & $75,57 \pm 18,4^{4}$ & $70,31 \pm 23,1^{\dagger ! !}$ \\
\hline Rol Físico (RF) & $89,74 \pm 27,3$ & $93,23 \pm 23,5^{z}$ & $85,71 \pm 31$ & $73,44 \pm 42,3^{2}$ \\
\hline Dolor Corporal (DC) & $80,96 \pm 23,1$ & $81,25 \pm 17,8$ & $77,50 \pm 22,9$ & $74,38 \pm 26,3$ \\
\hline Salud General (SG) & $75,38 \pm 23,4$ & $73,88 \pm 16,5$ & $73 \pm 20,2$ & $70,63 \pm 26,3$ \\
\hline Vitalidad (V) & $80,51 \pm 15,1$ & $79,58 \pm 17$ & $77,14 \pm 18$ & $72,42 \pm 22,2$ \\
\hline Función Social (FS) & $89,42 \pm 16,4$ & $87,76 \pm 19,5$ & $87,14 \pm 16,7$ & $89,06 \pm 15$ \\
\hline Rol Emocional (RE) & $89,74 \pm 28,8$ & $90,28 \pm 26,6$ & $90,48 \pm 27,5$ & $89,58 \pm 26,4$ \\
\hline Salud Mental (SM) & $89,74 \pm 12,8$ & $86,25 \pm 17,8$ & $85 \pm 18,8$ & $86,88 \pm 28$ \\
\hline Transición Salud (TS) & $78,85 \pm 23$ * & $74,48 \pm 25$ & $65,71 \pm 24,5^{\text {t }}$ & $68,75 \pm 21$ \\
\hline
\end{tabular}

${ }^{+}$Significancia estadística $p<0.05$ entre 6o-64 Vs 70-75 y 75 años y más

"! Significancia estadística p<0.05 entre 65-69 Vs 75 años y más

${ }^{z}$ Significancia estadística $p<0.05$ entre 65-69 Vs 75 años y más

${ }^{*}$ Significancia estadística $p<0.05$ entre 6o-64 Vs 70-75

En la tabla 1 se presentan los resultados del SF-36 y su comportamiento de acuerdo con los rangos de edad. Se encontró que la dimensión FF presentó diferencias significativas entre los rangos de 6o-64 años 70-74 años y 75 años y más, así como entre los rangos de edad de 65-69 años y el rango de 75 años y más ( $\mathrm{p}<0.05)$; además, el rango 6o64 años presentó el valor más alto. El RF mostró diferencias significativas $(\mathrm{p}<0.05)$ entre los rangos de edad de $65-69$ años y el de 75 años y más, siendo el rango 65-69 años donde se registra el mejor valor del RF. En la TS se encontró diferencias significativas entre los rangos de edad de 6o-64 años y 7074 años (p<0.05). En las demás dimensiones (DC, SG, V, FS, RE y SM) no se hallaron diferencias significativas ( $p>0.05$ ). Asimismo, importante comprender que el rango de edad de 6o-64 años es el que muestra los valores promedio más altos en la mayoría de las dimensiones de la calidad de vida. 


\section{Bienestar Social}

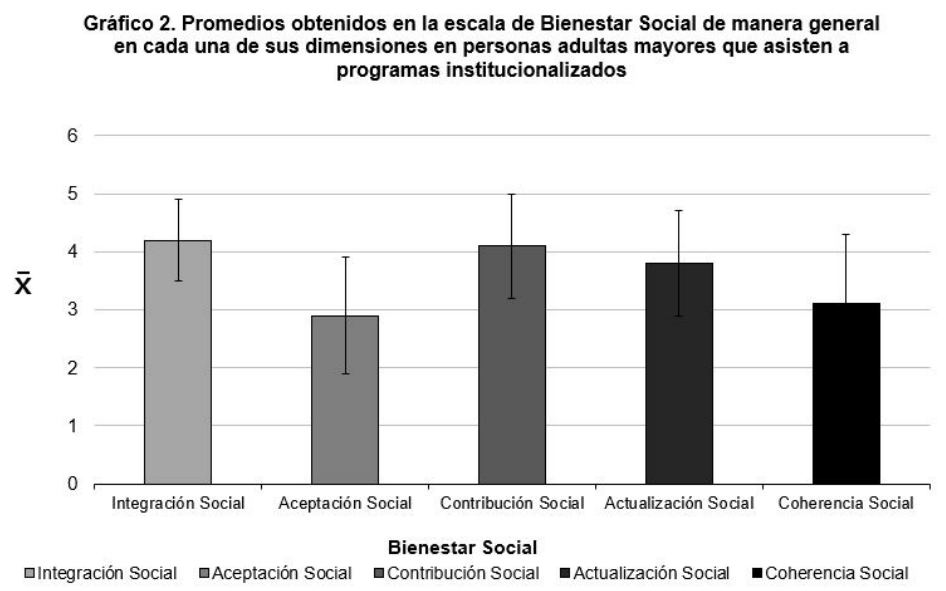

El gráfico 2 muestra los valores promedio obtenidos en cada percibidos con mayor impacto por la persona adulta mayor, no una de las sub-escalas de bienestar social. La integración social, así la aceptación social que obtuvo un valor promedio menor a 3.

la contribución social y la actualización social son factores

Tabla 2. Promedios obtenidos en la escala de bienestar social por rango de edad en personas adultas mayores evaluadas.

\begin{tabular}{lllll}
\hline & $60-64$ años & $65-69$ años & $70-74$ años & 75 y más años \\
& $\mathrm{N}=39$ & $\mathrm{~N}=48$ & $\mathrm{~N}=35$ & $\mathrm{~N}=16$ \\
& $\overline{\mathrm{x}} \pm \mathrm{DS}$ & $\overline{\mathrm{x}} \pm \mathrm{DS}$ & $\overline{\mathrm{x}} \pm \mathrm{DS}$ & $\overline{\mathrm{x}} \pm \mathrm{DS}$ \\
\hline Integración Social & $4,3 \pm .58$ & $4,14 \pm .68$ & $4,17 \pm .67$ & $4,26 \pm .95$ \\
Aceptación Social & $2,9 \pm .91$ & $2,9 \pm 1,1$ & $2,8 \pm 1,1$ & $2,75 \pm .82$ \\
Contribución Social & $4,22 \pm .77$ & $4,1 \pm .90$ & $4,01 \pm 1$ & $4,24 \pm .96$ \\
Actualización Social & $4,17 \pm .64^{\dagger}$ & $3,75 \pm .99^{\dagger}$ & $3,4 \pm .94 \dagger^{* !}$ & $4,03 \pm .79^{! !}$ \\
Coherencia Social & $3,4 \pm 1,1^{z}$ & $2,8 \pm 1,2^{z}{ }^{\natural}$ & $2,9 \pm 1,3^{*}$ & $3,7 \pm 1,4^{*}$ \\
\hline
\end{tabular}

${ }^{+}$Significancia estadística p<0.05 entre 6o-64 Vs 65-69 y 70-74

"! Significancia estadística p<0.05 entre 70-74 Vs 75 años y más

${ }^{z}$ Significancia estadística $p<0.05$ entre 6o-64 Vs 65-69s

* Significancia estadística p<0.05 entre 65-69 Vs 70-74 y 75 años y más

La tabla 2 indica que la actualización social mostró diferencias significativas entre el rango de edad de 6o-64 años versus 65-69 años y 70-74 años (p<0.05). En la dimensión coherencia social hubo diferencias significativas $(\mathrm{p}<0.05)$ entre los rangos de edad de 6o-64 versus 65-69 años. El rango de edad de 6569 años se diferenció con la categoría de edad 70-74 años y 75 años y más ( $\mathrm{p}<0.05)$. 


\section{Salud General}

Se describe a continuación los resultados reportados en cuanto a la percepción de salud general que manifestaron las personas analizadas.

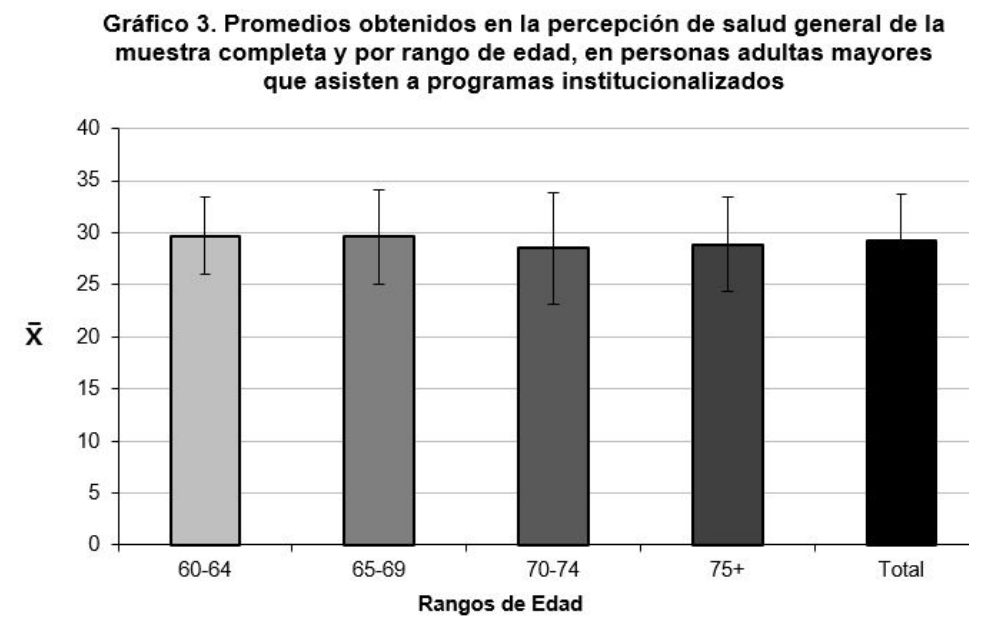

En el gráfico 3 se muestran los valores promedio de la percepción de salud por rango de edad de las personas participantes. El rango de edad de 6o-64 años el de mejor percepción de salud y el rango de 70-74 años presenta la percepción más baja de salud. Sin embargo, en la escala de Salud General no se observaron diferencias significativas ( $p>0.05$ ) entre los rangos de edad.

\section{Bienestar social y calidad de vida}

Tabla 3. Matriz de correlación entre la calidad de vida (SF-36) y las variables de la escala de bienestar social, en personas adultas mayores evaluadas.

\begin{tabular}{|c|c|c|c|c|c|c|c|c|c|}
\hline & \multicolumn{9}{|c|}{ Calidad de Vida SF 36} \\
\hline & $\begin{array}{l}\text { Función } \\
\text { física }\end{array}$ & $\begin{array}{l}\text { Rol } \\
\text { físico }\end{array}$ & $\begin{array}{l}\text { Dolor } \\
\text { corporal }\end{array}$ & $\begin{array}{l}\text { Salud } \\
\text { general }\end{array}$ & Vitalidad & $\begin{array}{l}\text { Función } \\
\text { social }\end{array}$ & $\begin{array}{l}\text { Rol } \\
\text { emocional }\end{array}$ & $\begin{array}{l}\text { Salud } \\
\text { mental }\end{array}$ & $\begin{array}{l}\text { Transición } \\
\text { salud }\end{array}$ \\
\hline \multicolumn{10}{|l|}{ Bienestar Social } \\
\hline Integración Social & $.21^{*}$ & & & $.24^{* *}$ & $.33^{* *}$ & $.23^{* *}$ & & $.36^{* *}$ & \\
\hline Aceptación social & & & & & $.19^{*}$ & & & & \\
\hline Contribución social & $.24^{* *}$ & & & $.28^{* *}$ & $.24^{\star *}$ & $.28^{* *}$ & & $.33^{* *}$ & $.19^{*}$ \\
\hline Actualización social & $.24^{* *}$ & & & $.19^{*}$ & $.19^{*}$ & $.19^{*}$ & & $.23^{\star *}$ & $.23^{* *}$ \\
\hline Coherencia social & & & & & & & & & \\
\hline
\end{tabular}

\footnotetext{
** La correlación es significativa al nivel de $\mathrm{p}<0.01$
}

* La correlación es significativa al nivel de $\mathrm{p}<0.05$ 
La tabla 3 expresa las correlaciones entre las dimensiones del cuestionario calidad de vida SF-36 y el de Bienestar Social. Se registraron correlaciones significativas entre la Integración Social y las dimensiones de Salud General, Vitalidad, Función Social y Salud Mental ( $\mathrm{p}<0.01)$ y con la Función Física $(\mathrm{p}<0.05)$. La dimensión Contribución Social se relacionó con los dominios de Función Física,
Salud General, Vitalidad, Función Social, Salud Mental ( $\mathrm{p}<0.01)$ y con Transición de Salud ( $\mathrm{p}<0.05$ ). Por su parte la Actualización Social se correlacionó positivamente con la Función Física, Salud Mental y Transición de Salud (p<0.01) y con la Salud General, Vitalidad y Función Social $(\mathrm{p}<0.05)$.

\section{Calidad de vida y salud general percibida}

Tabla 4. Matriz de correlación entre la Calidad de Vida (SF-36) y Salud General, en personas adultas mayores analizadas.

\begin{tabular}{|c|c|c|c|c|c|c|c|c|c|}
\hline & \multicolumn{9}{|c|}{ Calidad de Vida SF 36} \\
\hline & $\begin{array}{l}\text { Función } \\
\text { física }\end{array}$ & $\begin{array}{l}\text { Rol } \\
\text { físico }\end{array}$ & $\begin{array}{l}\text { Dolor } \\
\text { corporal }\end{array}$ & $\begin{array}{l}\text { Salud } \\
\text { general }\end{array}$ & Vitalidad & $\begin{array}{l}\text { Función } \\
\text { social }\end{array}$ & Rol emocional & $\begin{array}{l}\text { Salud } \\
\text { mental }\end{array}$ & $\begin{array}{l}\text { Transición } \\
\text { salud }\end{array}$ \\
\hline $\begin{array}{l}\text { Salud } \\
\text { general }\end{array}$ & $30^{* *}$ & $.19^{*}$ & $.28^{* *}$ & $.45^{* *}$ & $.51^{* *}$ & $.43^{* *}$ & $\cdot 31^{* *}$ & $.50^{\star \star}$ & $.24^{* *}$ \\
\hline
\end{tabular}

** La correlación es significativa al nivel de $\mathrm{p}<0.01$

* La correlación es significativa al nivel de $\mathrm{p}<0.05$

En la tabla 4 se muestra que la calidad de vida se correlacionó positivamente en cada una de sus dimensiones con la salud general percibida $(\mathrm{p}<0.01)$.

\section{Bienestar social y salud general}

Tabla 5. Matriz de correlación entre la escala de bienestar social salud general, en personas adultas mayores estudiadas.

\begin{tabular}{|c|c|c|c|c|c|}
\hline & \multicolumn{5}{|l|}{ Bienestar social } \\
\hline & Integración social & Aceptación social & Contribución social & Actualización social & Coherencia social \\
\hline Salud general & $.33^{* *}$ & & $34^{* *}$ & $.22^{* *}$ & $.21^{*}$ \\
\hline
\end{tabular}

** La correlación es significativa al nivel de $\mathrm{p}<0.01$

* La correlación es significativa al nivel de $\mathrm{p}<0.05$

Las dimensiones de bienestar social se correlacionaron de modo positivo con la percepción de salud ( $<<0.05$ ), excepto la Aceptación Social que no mostró correlación con la percepción de salud (p>0.05). 


\section{Discusión}

Los resultados de este estudio mostraron una interrelación entre el bienestar social, la salud y la calidad de vida en las personas adultas mayores que participan en programas institucionalizados donde realizan actividades lúdicas. En detalle, los datos revelan una buena calidad de vida de las personas adultas mayores evaluadas y al analizar los componentes de la calidad de vida, se debe indicar que, conforme avanza la edad de las personas evaluadas la función física va decreciendo, aspecto determinado por el grupo de edad de 6o-64 años, ya que este fue significativamente diferente de los otros. La percepción de una función física disminuida en las personas conforme avanza en su edad se relaciona con los cambios fisiológicos que sobrevienen del proceso natural de envejecimiento a pesar de que se sometan a programas de actividad física. En alusión a los resultados presentados, según expertos, la práctica de ejercicio físico diario, si bien es cierto retarda los efectos del envejecimiento, no los elimina del todo, por lo que es de esperar que a mayor edad, haya un deterioro de la función física en las personas (ACSM, 2018; Bushman, 2017).

Por su parte, el rol físico se relaciona con el proceso natural fisiológico del envejecimiento, ya que con el paso de los años cambia la apariencia, hay pérdida de masa muscular, masa ósea, disminución en la tasa metabólica, disminuye la capacidad de reacción, la función cerebral, las funciones del sistema inmune, la capacidad física y una serie de cambios endocrinos que van limitando el funcionamiento óptimo del organismo (Kaupuzs y Larins, 2008). En estas circunstancias, es probable que se presente un deterioro importante de la salud, lo que limita el quehacer diario de las personas y la percepción de su capacidad o funcionamiento físico se ve disminuida (Vilagut et al., 2005). Estos aspectos mencionados explican los resultados encontrados en cuanto al rol físico percibido, ya que, conforme avanza la edad hay una percepción de disminución significativa del rol físico.

En contraparte, los resultados encontrados reflejan que las personas participantes en este estudio no manifestaron niveles de dolor corporal importantes que limitaran su funcionalidad, situación que es explicada por científicos como un efecto positivo de mantenerse activo físicamente
(Landmark et al., 2011). Previas investigaciones demuestran que la actividad física diaria representa una estrategia de afrontamiento del dolor efectiva (Ambrose y Golightly, 2015; Rainville et al., 2004). En una investigación previa se encontró que las personas activas físicamente lograron reducir su sensación de dolor de manera significativa, mientras que quienes se mantuvieron inactivos mostraron niveles de dolor cada vez mayores según su condición ociosa; además las personas con niveles disminuidos de dolor mostraron mejores niveles de calidad de vida (Torre et al., 2008).

Para lograr una buena calidad de vida, es necesario poseer una salud general percibida adecuada y esta a su vez es un factor determinante en la capacidad funcional e independencia de las personas adultas mayores. La salud percibida es una variable de mayor impacto en el desempeño general de este grupo poblacional y de ahí la importancia de su evaluación. De este modo, en investigaciones desarrolladas en países como Perú, Dinamarca, Estados Unidos, Francia, Japón, México, Australia, Holanda e Italia, muestran que las personas adultas mayores poseen una buena salud general (Coronado et al., 20o9; Kaupuzs y Larins, 2008). Por el contrario, en estudios realizados con población adulta mayor costarricense, se encontró que un alto porcentaje de las personas analizadas manifestaron tener su salud en malas o muy malas condiciones (Cáceres, 2004; OPS, 2004).

Aunado al sentimiento de salud adecuado, se encuentra la vitalidad la cual es fundamental para enfrentar la vida con entusiasmo y energía. En este estudio los resultados indicaron una vitalidad positiva, pero conforme aumenta la edad de los participantes, la percepción de vitalidad es menor, aunque no a un nivel significativo. Aspecto que debe ser considerado para una calidad de vida plena. La vitalidad fue analizada en un estudio previo en Colombia, determinando que las personas adultas mayores percibían una vitalidad moderada, y que la calidad de vida estaba deteriorada, lo que refuerza la importancia de mantener niveles de vitalidad elevados en este grupo poblacional en particular (Ramírez et al., 2008). 
Otro componente esencial de la calidad de vida es la función social, en detalle un componente determinante para las personas adultas mayores, ya que la participación social conectada a relaciones sociales positivas generan un sentimiento de pertenencia que influye en la calidad de vida, situación que se confirmó en los resultados obtenidos en esta investigación. En previos estudios se reportó que la dimensión social es un fuerte determinante de la calidad de vida, muchos problemas de salud, desórdenes psicológicos y otros aspectos se derivan de una participación social negativa; además, la falta de relaciones sociales positivas fue uno de los factores relacionados con la aparición de enfermedades degenerativas y por ende, con el consecuente deterioro de la calidad de vida (Levasseur et al., 2004; Unger et al., 1999; Seeman , Chen 2002; Zimmer y House 2003).

Por su parte, los resultados mostraron que el rol emocional fue la dimensión con mayor fortaleza de manera general y por rango de edad, indicando una mejor auto-estima y seguridad emocional de los participantes. Estos resultados concuerdan con los presentados en un estudio previo realizado en Perú y España, donde se reportó que el rol emocional fue la dimensión que mostraba promedios más elevados (Coronado et al., 2009). En contraparte, en Colombia se encontró un rol emocional deteriorado, las personas adultas mayores reportaron promedios muy bajos en esta dimensión, lo que indicó una situación social anormal, generadora de una mala calidad de vida (Ramírez et al., 2008). Es importante comprender que el bienestar emocional se relaciona con la inactividad física y podría, por tanto, estar más disminuido en aquellas personas con niveles bajos de actividad física y salud (Mella et al., 2004). El ejercicio físico tiene como beneficio adicional la mejora del bienestar general y lleva a las personas a estilos de vida más saludables, con mayor seguridad y autoestima (Chávez et al., 2018), esto hace que su función emocional mejore considerablemente, por lo que no es de extrañar que las personas con estilos de vida sedentarios manifiesten estados emocionales deteriorados (Prentice, 2015).

La fortaleza mental es un determinante importante de la calidad de vida y que podría ser fomentado a través de una vida más activa físicamente, lo que se relaciona con los resultados encontrados en el presente análisis, destacando que la salud mental presentó valores altos, indicando así que el hecho de pertenecer a un grupo organizado de actividad lúdica no solo mejora los aspectos biológicos de las personas adultas mayores, sino que también interviene positivamente sobre los procesos mentales. Acorde con esta posición, en un estudio previo se reportaron resultados promedio bajos en esta dimensión en personas sedentarias, lo que generó riesgos adicionales de presentar psicopatologías como depresión, ansiedad, pérdida del auto-control y el detrimento del bienestar general (Ramírez et al., 2008).

Por último, la transición de salud surge como un factor que se relaciona con los cambios acaecidos de un año a otro en el estado de salud. Para el Colegio Americano de Medicina Deportiva (ACSM, 2018), la aparición de enfermedades se hace más frecuente en edades más avanzadas y esto lleva a dificultades mayores para participar activamente en los programas de ejercicio, disminuye la capacidad de ejecución y, por supuesto, disminuyen los beneficios generados. En los resultados se pudo observar que el rango de edad más joven percibió una mejor transición de salud en comparación con los de edad más avanzada; es decir, los cambios en la salud de un año a otro marcan diferencia y las personas sienten con mayor severidad el paso de los años y cómo se va comprometiendo la salud que se disfruta, a pesar de que se trata de personas activas físicamente.

Por otra parte, el apoyo social con reconocido impacto sobre el bienestar general de la persona adulta mayor, permite la creación y el mantenimiento de redes de apoyo social, ofrece la oportunidad de mejorar y conservar el estatus de salud y el bienestar a pesar del paso de los años; este apoyo social es un valor agregado que se asocia con la práctica de actividad física en grupos organizados, ya que las personas se relacionan e interactúan constantemente (Hellstrom et al., 2004, White et al., 2009, Vera 2013). El presente estudio mostró que las personas evaluadas se sienten integradas socialmente, lo que se percibe como una fortaleza, generando una protección adicional contra situaciones de riesgo para la salud y la calidad de vida. El hecho de ser parte de relaciones sociales de calidad es tan importante como el efecto protector que produce 
el mismo ejercicio físico (Glass et al., 1999; Unger et al., 1999). Se ha demostrado que integrarse y participar en actividades sociales que requieren de ningún o poco esfuerzo físico, pueden resultar en una disminución del riesgo de mortalidad y este beneficio es tan importante como el obtenido en aquellas actividades que requieren esfuerzo físico más intenso. Esta situación es relevante, ya que personas con algún nivel de dependencia funcional y que por su condición no pueda realizar esfuerzos físicos extenuantes, se pueden beneficiar con la simple participación en un programa de actividad física (Laguado et al., 2017; Berkman et al., 2004; Glass et al., 1999; Holtzman et al., 2004). Por su parte, con frecuencia se señala el efecto de la integración y la participación social sobre la salud (Berkman et al., 2000; Glass et al., 1999; Holtzman et al., 2004; Unger et al., 1999; Otero et al., 2006), argumentando que las personas que tienen dificultades para integrarse a la sociedad y se aíslan, tienen mayores riesgos de desarrollar enfermedades y aumentan su riesgo de mortalidad temprana.

Otro factor importante a considerar en el bienestar social de la persona adulta mayor es la aceptación social, la cual mostró los valores más bajos y aunque no fue afectada significativamente, es un hecho que concierne a la sociedad actual. La aceptación social se relaciona con el sentido de pertenencia al grupo y el disfrute de ella en las cualidades de confianza, aceptación y actitudes positivas hacia los demás, así como la aceptación de los aspectos positivos y negativos propios (Corey, 1998). Este fenómeno podría estar relacionado con la presencia de algún grado de desconfianza e intranquilidad, provocado por la inseguridad social, la pérdida de valores, el abandono y otras situaciones que están sucediendo con la sociedad actual y que además, esto podría generar problemas de salud mental (Berkman et al., 2004; Glass et al., 1999; Unger et al., 1999; Puga et al., 2007). De este modo, la pertenencia a un grupo o red social (grupo de amigos, de trabajo, ejercicio) produce efectos positivos para la salud cognitiva; una persona adulta mayor que pertenece a un grupo y se siente segura en él, mantiene un buen estado emocional y de salud duradero (Holtzman et al., 2004; Corey, 1998; Ferreira et al., 2017).
Según lo planteado, la pertenencia a un grupo indica una contribución social que se relaciona con el sentimiento de utilidad propio para la sociedad, que se es miembro de la sociedad y se tiene algo importante que ofrecer ella (Corey, 1998; Levasseur et al., 2004). Es importante resaltar que las personas evaluadas indicaron que su contribución a la sociedad es alta, lo que podría generar efectos positivos para la salud y la calidad de vida. Según un reporte previo, la participación social produce efectos positivos para la salud, contribuye a disminuir la depresión, aumenta la salud percibida y disminuyen los problemas funcionales e instrumentales que aparecen en las primeras etapas de la discapacidad (Puga et al., 2007).

Además de la participación, la actualización social juega un papel importante en el bienestar general de la persona adulta mayor. Indicando que las personas confían en el futuro de la sociedad, en su potencial de progreso y de desarrollo, en su capacidad para producir bienestar (Corey, 1998). Esta situación se relaciona con el entendimiento del dinamismo de la sociedad y de las instituciones, esto permite el crecimiento social y personal; la gente que goza de más salud mental, tiene confianza en el futuro social, siente que puede crecer y desarrollarse y que esto le puede generar bienestar (Blanco y Díaz, 2005).

Como un último aspecto del bienestar social, es importante resaltar la coherencia social, demostrando que la persona adulta mayor comprende bien lo que sucede con la sociedad, entiende lo que pasa alrededor de cada quién, entiende la lógica del porqué suceden cosas que no están dentro de lo normal; a pesar de la complejidad del mundo, no es difícil comprenderlo. Para explicar esta percepción, algunos investigadores indican que las personas activas se interesan por conocer y comprender lo que sucede a su alrededor, además de encontrar sentido y lógica a los acontecimientos (Blanco y Díaz, 2005). El bienestar social es un componente esencial para mantener un buen estatus psicológico y una buena salud general (Mella et al., 2004). 
Para comprender la realidad en cuanto a la salud general percibida y su importancia, se debe mencionar los resultados de diferentes investigaciones. Por ejemplo, en el I Informe Estado Situación de la Persona Adulta Mayor (Fernández y Robles, 2008) se mostró que el 56\% de las personas adultas mayores que vivían fuera de la Gran Área Metropolitana de Costa Rica y el 41\% de los que vivían en ella, percibieron su salud como regular o mala. Mientras tanto, el $47 \%$ de las personas encuestadas manifestaron que su salud era regular o mala. Para el caso de Cuba, se reportó que solamente el 39,6\% de las personas adultas mayores coincidieron en que su salud es positiva, o sea, un 6o,4\% reportaron su salud como deficiente (Fernández et al., 200o). En Letonia se encontró que el 19,3\% de las personas manifestaron tener su salud deteriorada, el 9,7\% indicaron que su salud era buena y el 71\% indicó su salud como normal o en el promedio (Kaupuzs y Larins, 2008). Por su parte, en Costa Rica, en un estudio con grupos no organizados en el año 2000, se encontró que el 22,7\% de las personas participantes reportaron su salud como buena, o sea un estado sano, mientras que el 77,3\% indicaron una salud deteriorada (Cáceres, 2004). En otro estudio comparativo entre países, se reportó que en Costa Rica se evidenció una salud autopercibida mala o regular significativamente más elevada entre las personas adultas mayores en comparación con poblaciones de Inglaterra y España (Puga et al., 2007). Los resultados del presente estudio indicaron una salud percibida buena, lo que podría ser atribuido a la participación de la persona adulta mayor en programas de actividad física y es un aliciente para que esta población sea motivada a realizar más actividad lúdica de calidad y en compañía de sus pares.

En conclusión, las personas que asisten a programas institucionalizados donde realizan actividad física en forma de caminata, baile, aeróbicos y otras actividades lúdicas, poseen un bienestar social elevado, relacionado con una buena salud percibida y una buena calidad de vida. En detalle, la participación en programas organizados de actividad física le permite a la persona adulta mayor desarrollar un adecuado bienestar social mostrado desde niveles bajos de dolor corporal, una actitud positiva ante la función social, el rol emocional y salud mental. El bienestar social percibido permite la integración y la participación de las personas adultas mayores en la sociedad; su aporte a la sociedad es de gran utilidad, tienen mucho que ofrecer a las nuevas generaciones motivo por el cual se sienten importantes para el desarrollo social. Esta percepción de bienestar en la persona adulta mayor promueve aspectos determinantes como la calidad de vida y la salud. Sin embargo, un aspecto a considerar es que, conforme avanza su edad, la percepción que se tiene sobre estas variables va disminuyendo, afectando negativamente el estado integral. Según los hallazgos presentados, se deben desarrollar programas de mediación que atiendan todas las particularidades de la persona adulta mayor a nivel nacional e internacional, ya que intervenciones que consideran los factores fisiológicos, los aspectos psicológicos y sociales, van a producir un mayor impacto sobre el bienestar social, la salud y la calidad de vida de este grupo poblacional. Con una intervención efectiva y oportuna para desarrollar el bienestar social, la salud y la calidad de vida, los beneficios posiblemente se postergarán de forma preventiva hasta edades avanzadas.

\section{Bibliografía}

ACSM, A. C. o. S. a. M. (2018). ACSM's guidelines for exercise testing and prescription.

Ambrose, K. R. y Golightly, Y. M. (2015). 'Physical exercise as non-pharmacological treatment of chronic pain: Why and when', Best Practice \& Research Clinical Rheumatology, 29(1), 120-130.

Berkman, L. F., Glass, T., Brissette, I. y Seeman, T. E. (2000). 'From social integration to health: Durkheim in the new millennium', Soc Sci Med, 51(6), 843-57.

Berkman, L. F., Melchior, M., Chastang, J. F., Niedhammer, I., Leclerc, A. y Goldberg, M. (2004). 'Social integration and mortality: a prospective study of French employees of Electricity of France-Gas of France: the GAZEL Cohort', Am J Epidemiol, 159(2), 167-74. 
Blanco, A. y Díaz, D. (2005). 'El bienestar social: Su concepto y medición. [Social well-being: Theoretical structure and measurement.]', Psicothema, 17(4), 582-589.

Bushman, B. (2017). ACSM's Complete Guide to Fitness \& Health., Second edition ed., Champaign, IL.: Human Kinetics.

Cáceres, R. I. (2004). 'Estado de salud, físico y mental de los adultos mayores del área rural de Costa Rica, 200o', Poblacion y Salud en Mesoamerica, 1(2).

Carbonell, A., Aparicio, V. A. y Delgado, M. (2009). 'Efectos del envejecimiento en las capacidades físicas: implicaciones en las recomendaciones de ejercicio físico en personas mayores', RICYDE. Revista Internacional de Ciencias del Deporte, V(17), 1-18.

Chávez, M. E., Bautista, A., García, D. A., Fuentes, M., Ogarrio, C. E., Montaño, E. E. y Hoyos, G. (2018). 'La aplicación de un programa de intervención para el beneficio de la salud física y emocional en mujeres adultas mayores en Hermosillo, Sonora', MHSalud, 15, 39-56.

Collazo, M. I., Calero, J. L., Ramos, R., Toledo, H., Morón, D., Pariente, T. G. y Valdés, R. (2017). 'Significados del envejecimiento desde la perspectiva de los ancianos', Revista Habanera de Ciencias Médicas, $16,657-665$.

Collins, K., Rooney, B. L., Smalley, K. J. y Havens, S. (2004). 'Functional fitness, disease and independence in community-dwelling older adults in western Wisconsin', WMJ, 103(1), 42-8.

Corey, M. K. (1998). ‘Social Well-Being', Social Psychology Quarterly, 61(2), 121-140.

Coronado, J. M., Díaz, C., Apolaya, M. A., Manrique, L. M. y Arequipa, J. P. (2009). 'Percepción de la calidad de vida relacionada con la salud del adulto mayor residente en la ciudad de Chiclayo', Acta Médica Peruana, 26, 230-238.
Fernández, N., Clúa, A. M., Báez, R. M., Ramírez, M. y Prieto, V. (200o). 'Estilos de vida, bienestar subjetivo y salud de los ancianos', Revista Cubana de Medicina General Integral, 16, 6-12.

Fernández, X. y Robles, A. (2008). I Informe estado de situación de la persona adulta mayor en Costa Rica, San José, Costa Rica: Universidad de Costa Rica.

Ferreira, M. C. G., Tura, L. F. R., Silva, R. C. D. y Ferreira, M. A. (2017). 'Social representations of older adults regarding quality of life', Rev Bras Enferm, 70(4), 8o6-813.

Gallegos, K., Garcia, C., Duran, C. A., Flores, Y. N. y Salmeron, J. (2009). 'Relationship between social support and the physical and mental wellbeing of older Mexican adults with diabetes', Rev Invest Clin, 61(5), 383-91.

García, C. V. (1999). 'Manual para la utilización del cuestionario de salud general de Goldberg: Adaptación cubana', Revista Cubana de Medicina General Integral, 15, 88-97.

Glass, T. A., Mendes de León, C., Marottoli, R. A. y Berkman, L. F. (1999). 'Population based study of social and productive activities as predictors of survival among elderly Americans', British Medical Journal, 319, 478-483.

Hellstrom, Y., Andersson, M. y Hallberg, I. R. (2004). 'Quality of life among older people in Sweden receiving help from informal and/or formal helpers at home or in special accommodation', Health Soc Care Community, 12(6), 504-16.

Holtzman, R. E., Rebok, G. W., Saczynski, J. S., Kouzis, A. C., Wilcox Doyle, K. y Eaton, W. W. (2004). 'Social network characteristics and cognition in middleaged and older adults', J Gerontol B Psychol Sci Soc Sci, 59(6), P278-84. 
Kaupuzs, A. y Larins, V. (2008). 'Self-perceived physical and Health condition of older persons (aged 6575)', Acta Kinesiologiae Universitatis Tartuensis, 13, 39-50.

Laguado, E., Camargo, K., Campo, E. y Martín, M. (2017). 'Funcionalidad y grado de dependencia en los adultos mayores institucionalizados en centros de bienestar', Gerokomos, 28, 135-141.

Landmark, T., Romundstad, P., Borchgrevink, P. C., Kaasa, S. y Dale, O. (2011). 'Associations between recreational exercise and chronic pain in the general population: Evidence from the HUNT 3 study', PAIN R, 152(10), 2241-2247.

Levasseur, M., Desrosiers, J. y Noreau, L. (2004). 'Is social participation associated with quality of life of older adults with physical disabilities?', Disabil Rehabil, 26(20), 1206-13.

Lopez, E., Banegas, J. R., Graciani, A., Gutierrez, J. L., Alonso, J. y Rodriguez, F. (2003). '[Populationbased reference values for the Spanish version of the SF-36 Health Survey in the elderly]', Med Clin (Barc), 120(15), 568-73.

Mella, R., González, L., D’Appolonio, J., Maldonado, I., Fuenzalida, A. y Díaz, A. (2004). 'Factores Asociados al Bienestar Subjetivo en el Adulto Mayor', Psykhe (Santiago), 13, 79-89.

Morales, F. (2015). 'El envejecimiento en Costa Rica: una perspectiva actual y futura', Acta Médica Costarricense, 57(2), 74-79.

OPS, O. P. d. l. S. (2004). La Salud de las Personas Adultas Mayores en Costa Rica, 56, San José, Costa Rica: Ministerio de Salud.

Ortiz, J. B. y Castro, M. (2009). 'Bienestar psicológico de los adultos mayores, su relación con la autoestima y la autoeficacia: Contribución de enfermería', Ciencia y enfermería, 15, 25-31.
Otero, A., Zunzunegui, M. V., Béland, F., Rodríguez, A. y García, M. (2006). Relaciones sociales y envejecimiento saludable, España: Fundación BBVA.

Penninx, B. W., Leveille, S., Ferrucci, L., van Eijk, J. T. y Guralnik, J. M. (1999). 'Exploring the effect of depression on physical disability: longitudinal evidence from the established populations for epidemiologic studies of the elderly', Am J Public Health, 89(9), 1346-52.

Prentice, W. (2015). Get Fit, Stay Fit, Seventh Edition ed., United States: F. A. Davis Company.

Puga, D., Rosero, L., Glaser, K. y Castro, T. (2007). 'Red social y salud del adulto mayor en perspectiva comparada: Costa Rica, España e Inglaterra', Poblacion y Salud en Mesoamerica, 5(ESP).

Rainville, J., Hartigan, C., Martinez, E., Limke, J., Jouve, C. y Finno, M. (2004). 'Exercise as a treatment for chronic low back pain', The Spine Journal, 4(1), 106-115.

Ramírez, R., Agredo, R. A., Jerez, A. M. y Chapal, L. Y. (2008). 'Calidad de Vida y Condiciones de Salud en Adultos Mayores no Institucionalizados en Cali, Colombia', Revista de Salud Pública, 10, 529-536.

Rodríguez, M. (2008). 'La percepción de la persona adulta mayor en la sociedad ramonense actual', Pensamiento Actual, 8(10-11), 31-39.

Salinas, A., Marnrique, B. y Téllez, M. M. (2008). 'Redes de apoyo social en la vejez: adultos mayores beneficiarios del componente para Adultos Mayores del Programa Oportunidades', in III Congreso de la Asociación Latinoamericana de Población, Córdoba, Argentina,

Schwartzmann, L. (2003). 'Calidad de vida relacionada con la salud: Aspectos conceptuales', Ciencia $y$ enfermería, 9, 09-21. 
Seeman, T. y Chen, X. (2002). 'Risk and protective factors for physical functioning in older adults with and without chronic conditions: MacArthur Studies of Successful Aging', J Gerontol B Psychol Sci Soc Sci, 57(3), S135-44.

Torre, F., Martín, J., Callejo, A., Gómez, C., La Torre, S., Esteban, Vallejo, R. M. y Arizaga, A. (2008). 'Calidad de vida relacionada con la salud y estrategias de afrontamiento ante el dolor en pacientes atendidos por una unidad de tratamiento del dolor', Revista de la Sociedad Española del Dolor, 15, 83-93.

Unger, J. B., McAvay, G., Bruce, M. L., Berkman, L. y Seeman, T. (1999). 'Variation in the impact of social network characteristics on physical functioning in elderly persons: MacArthur Studies of Successful Aging', J Gerontol B Psychol Sci Soc Sci, 54(5), S245-51.

Vera, M. (2013). 'Significado de la calidad de vida del adulto mayor para sí mismo y para su familia', Anales de la Facultad de Medicina, 68(3), 284-290.

Vilagut, G., Ferrer, M., Rajmil, L., Rebollo, P., Permanyer, G., Quintana, J. M., Santed, R., Valderas, J. M., Ribera, A., Domingo, A. y Alonso, J. (2005). '[The Spanish version of the Short Form 36 Health Survey: a decade of experience and new developments]', Gac Sanit, 19(2), 135-50.

White, A. M., Philogene, G. S., Fine, L. y Sinha, S. (2009). 'Social support and self-reported health status of older adults in the United States', Am J Public Health, 99(10), 1872-8.

Zimmer, Z. y House, J. S. (2003). 'Education, income, and functional limitation transitions among American adults: contrasting onset and progression', Int J Epidemiol, 32(6), 1089-97. 